\title{
Optimal Performance Analysis Enabling OSPF and BGP in Internal and External WAN
}

\author{
K. RamKumar ${ }^{1}$, S. Raj Anand ${ }^{2}$ \\ ${ }^{1,2}$ VelTechMultiTech Dr. Rangarajan Dr. Sakunthala Engineering College, Chennai
}

\begin{abstract}
:-
Routing Iprotocols determines the shortest path to transfer the data from one host to another and specify how communication takes place between each routers. There are different classes of routing protocols are available in different environments such as intra and inter Autonomous System (AS).A routing protocol can be static or dynamic as well as link state and path vector. We will focus on Open Shortest Path First $(O S P F)$ and Border Gateway Protocol (BGP), OSPF and BGP protocols are dynamic in nature. BGP is an inter-autonomous system routing protocol and OSPF is an intra-a utonomous system routing protocol. We can analyze the performance of Wide Area Network (WAN) by enabling the Interior Gateway Protocol (IGP) and Exterior Gateway Protocol (EGP) of the network and ensure the Quality of Service $(Q o S)$.
\end{abstract}

\section{Keywords}

Open Shortest Path First, Border Gateway Protocol, Wide Area Network

\section{Introduction}

This study is a comparison of two routing protocols proposed for Wide area network. Routing protocols are the backbone of computer networks. We have to create different Autonomous system (AS) with multiple nodes using network topology. Each node in the network is called routers, forwarding data packets for other nodes. We can implement the routing protocols such as OSPF (Open Shortest Path First) within the autonomous system and BGP (Border Gateway Protocol) for connecting multiple autonomous systems. With the routing protocols we can analyze the routing and performance of the internal and external wide area network. Comparing OSPF protocols with the other IGP routing protocols such as IS-IS, Routing Information Protocol (RIP) and EIGRP [1]. Comparing the IGP and IBGP routing protocols by manipulating the metrics such as Administrative distance, convergence, delay, Maximum Transmission Unit (MTU) and bandwidth they can found best route [2]. By using simulation software they can analyze the performance of a network by manipulating the metrics of the EIGRP and OSPF routing protocols [3]. Creating an efficient network design by investigating the two protocols within the inter domain and intra domain by using OSPF and BGP [4]. To implement security in BGP address by implementing the systemic and operational design for the local network and internet routing infrastructure with different Autonomous System (AS) [5].Examine the inter domain routing information exchanged in networks between the backbone service providers at public internet exchange points for routing flops. [6]. Comparison of three wireless mobile ad-hoc 
network routing protocols like, Destination Sequenced Distance Vector (DSDV), Ad-hoc on Demand Distance Vector (AODV) and Dynamic Source Routing (DSR) [7].

\section{Related Work}

A Several researchers have done the routing and performance analysis of Wide Area Network (WAN) [1] by using different performance metrics of routing protocols. They have used different network simulators for this purpose. Routing Information Protocol (RIP) can be implemented in Wide Area Network for make communication between routers. Due to the limitations of the Routing Information Protocol (RIP) hop count WAN cannot be as much effective in Figure 1.

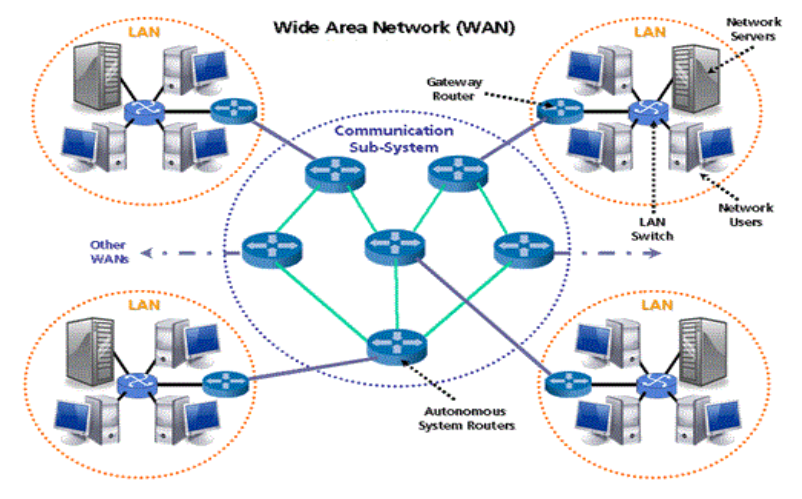

Figure 1: Wide Area Network using RIP

\section{Network Protocol Simulation Models}

The network simulation protocols include: OSPF and BGP.OSPF is a link-state Interior Gateway Protocol (IGP) designed to run in intra Autonomous System. Each OSPF router maintains an identical database describing the Autonomous System (AS) of the network's topology. From that identical database, a routing table is calculated by constructing a shortest-path tree using Dijkstra's algorithm. OSPF independently recalculates routes quickly if any changes happened in the topological. The only exception is that Network LSAs were not modeled, and so the topology was not configured with stub networks. External nodes were defined by using BGP (Border Gateway Protocol) outside the current Autonomous System (AS). Internal peers were fully connected to all BGP routers in a given Autonomous System (AS).

\subsection{OSPF (Open Shortest Path First)}

Open Shortest Path First (OSPF) is a link-state dynamic routing protocol for Internet Protocol (IP) networks. OSPF uses a link state routing algorithm, and operates within a single Autonomous System (AS).OSPF use Hello protocol to keep on updating the neighbor routers by sending hello packet for every 10 seconds. If the hello packet is not seen within Dead time interval (40 seconds) then the source router assumes the neighbor router was dead and status is send to other neighbors. 


\subsubsection{OSPF Distance, Metrics and Areas}

OSPF uses the default Administrative Distance as 110 for prioritizing the routing protocol which is implemented in the network. OSPF uses cost as their metrics which is used to calculate the shortest path among the neighbor routers, the cost can be calculated by inverse of the interface's bandwidth. However, which interface does OSPF uses to calculate its metric? Is it the neighbor's inbound interface or its outbound interface? or both? The multi-area OSPF are shown in Fig 2.

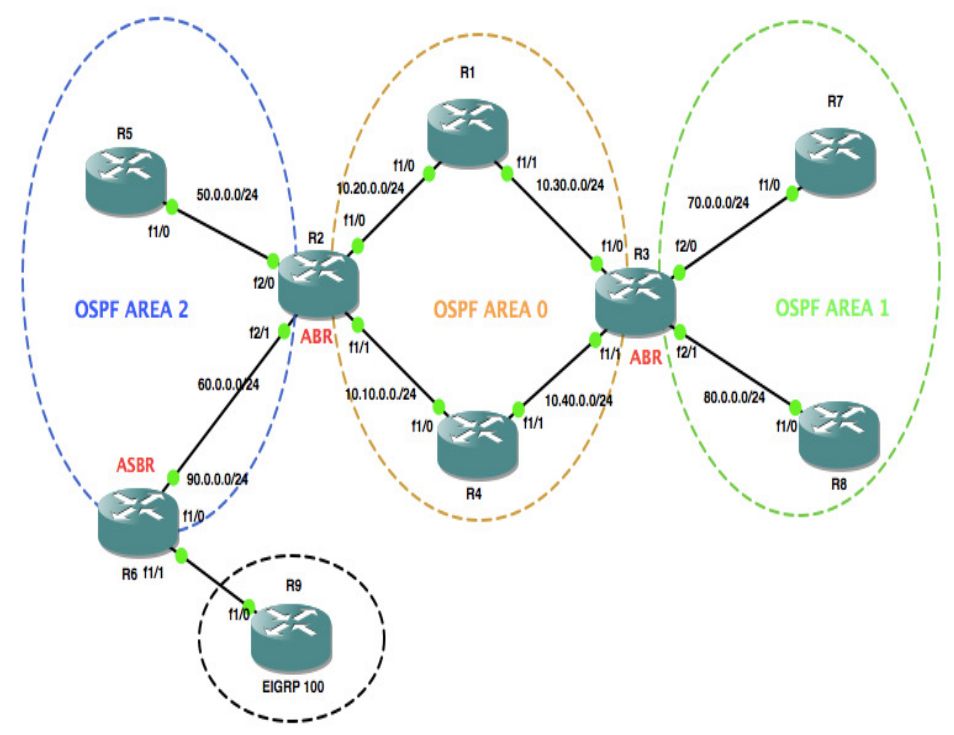

Figure 2: OSPF Multi Areas

\subsubsection{Link-State Advertisement}

Link-State Advertisement (LSA) is a basic communication mechanism of the OSPF routing protocol. It advertises the router's local routing topology to all the available local routers in the same OSPF area. OSPF is mainly designed for scalability in the network, so some LSAs are not flooded out on all the available interfaces, but only on those that are belong to the appropriate area in Fig 3. With the LSA the detailed information can be kept locally, while the summary information is flooded to the all the remaining available network. 


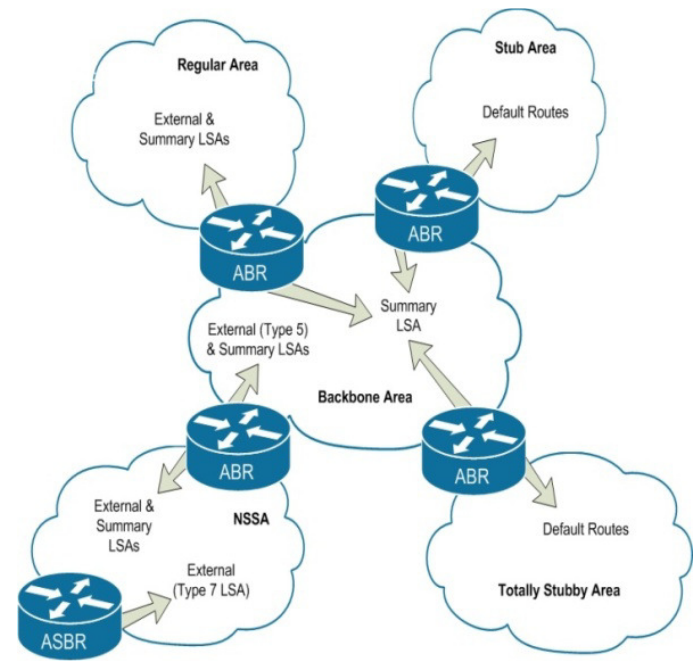

Figure 3: OSPF LSA

\subsection{BGP (Border Gateway Protocol)}

Border Gateway Protocol (BGP) is a only standardized Exterior Gateway Protocol (EGP) designed to exchange the routing and reach ability information of the destination node between multiple Autonomous Systems (AS) over the Internet. The protocol is classified as a path vector protocol, but is sometimes also called as a distance vector routing protocol. The Border Gateway Protocol (BGP) does not use the Interior Gateway Protocol (IGP) metrics, but it makes routing decisions based on paths, network policies and rule-sets configured by a network administrator. The Border Gateway Protocol plays a major role in the overall operation of the Internet and is involved in making core routing decisions.

Border Gateway protocol is the most widely used Exterior Gateway Protocol (EGP) by the ISP (Internet Service Provides) because BGP allows fully decentralized routing. When BGP runs between two peers in same Autonomous System (AS) which is called as IBGP (Interior Border Gateway Protocol) and when it runs between two different Autonomous Systems it is called as EBGP (Exterior Border Gateway Protocol).Routers on the border of one Autonomous System to exchanging information with another AS are called border or edge routers

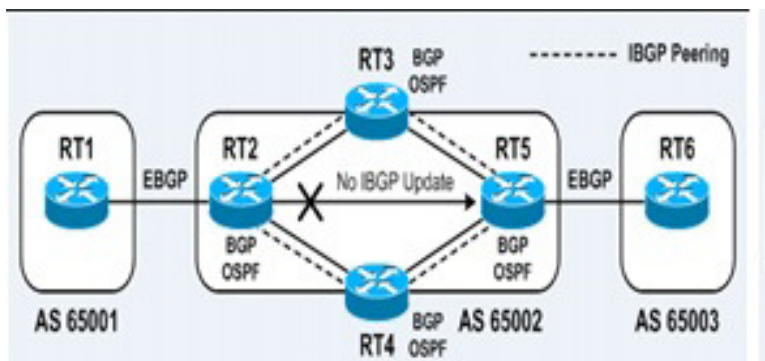

Figure 4: BGP with no routing update

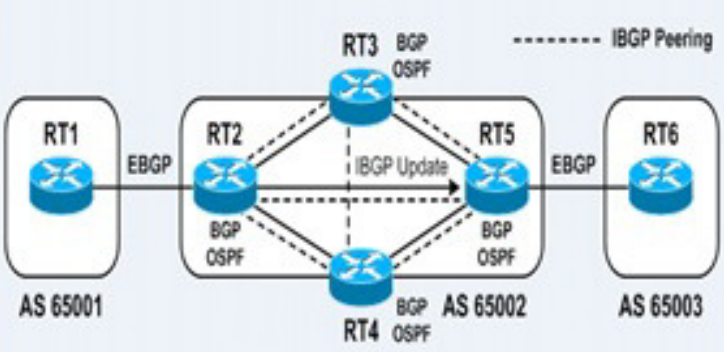

Figure 5: BGP with routing update 


\subsubsection{Operations of BGP}

The neighbors of BGP are called as peers. Among all the routing protocols, BGP is uniquely uses TCP as their transport protocol on port 179. For every 30 seconds BGP speakers send 19bytes of keep-alive messages to maintain the connection. BGP which runs between two peers in same autonomous system are called as iBGP (Interior Border Gateway Protocol) and BGP which runs in inter autonomous system are called as eBGP (Exterior Border Gateway Protocol). A router on the boundary of different autonomous systems was share the information between each other was called edge router shown in Figure 6.

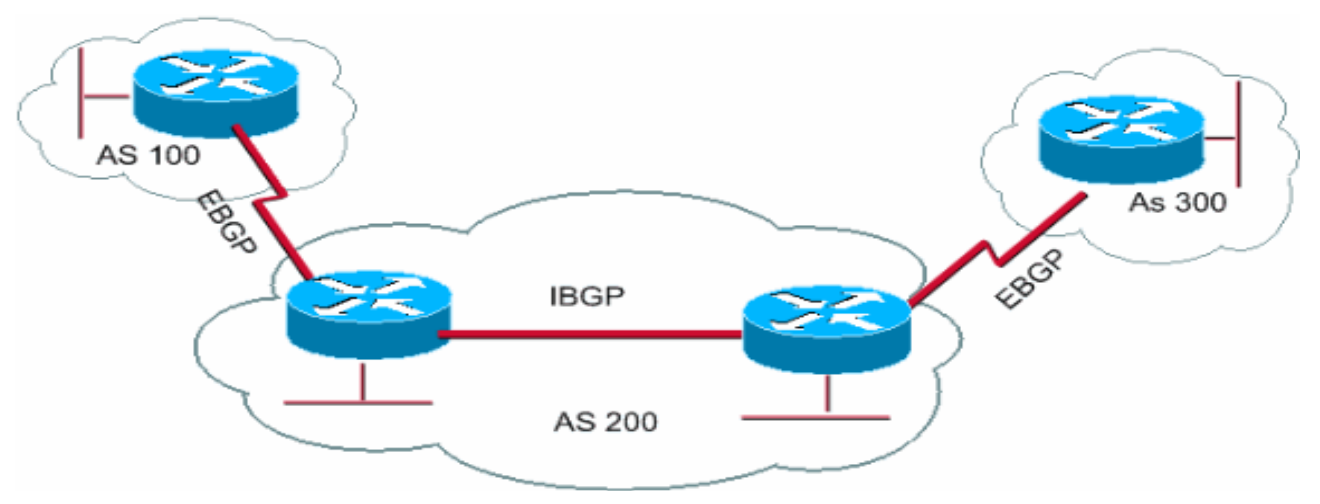

Figure 6: iBGP and eBGP

\section{Wide Area Network using OSPF and BGP Protocols}

Implemented in WAN (Wide Area Network) and also implemented in Inter Autonomous System Routing protocols like,

- $\quad$ OSPF(Open Shortest Path First)

- BGP (Border Gateway Protocol)

We have to modify all the metrics of the routing protocol using IPv4 technique.

By implementing the OSPF protocol within the Autonomous System (AS) or WAN (Wide Area Network) and modifying the metrics of OSPF (Open Shortest Path First) protocol such as. Cost, reliability, bandwidth and delay we can obtain the optimal performance in the Internal WAN.

We can use BGP (Border Gateway Protocol) for connect more than one Autonomous System (AS) and make communication among the network (Wide Area Network). By analyzing the internal and external WAN we can find out the optimal performance of both internal and external Wide Area Network. 
International Journal of Control, Automation, Communication and Systems (IJCACS), Vol.1, No.1, January 2016

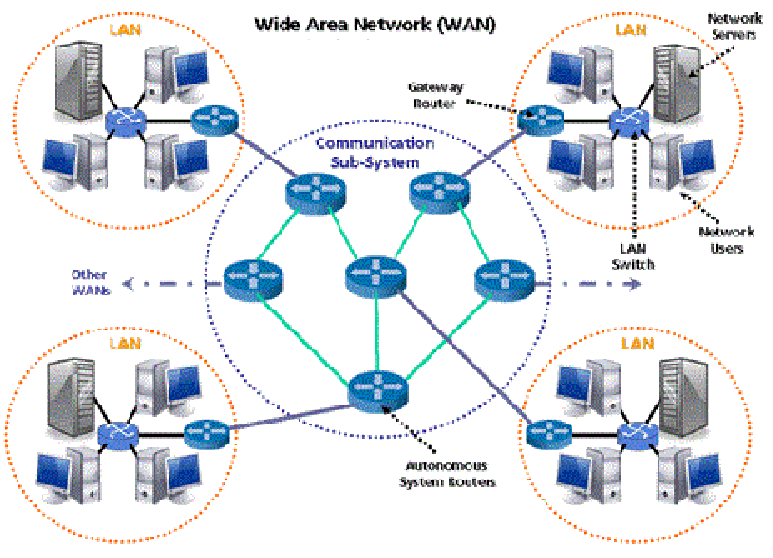

Figure 7: WAN using OSPF and BGP

\section{Result and Discussion}

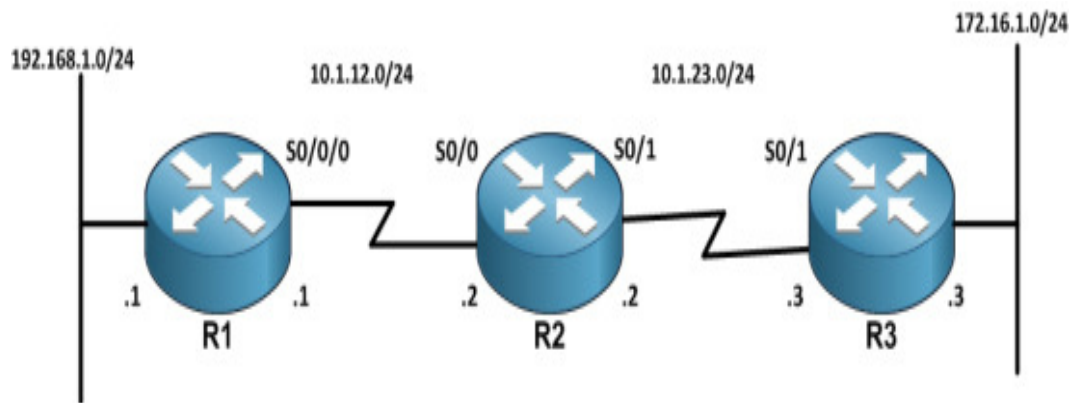

Figure 8: OSPF with Metrics

The system was configured with IP addresses and enabled OSPF for all the routers. Here I focus on R3's LAN, 172.16.1.0/24, metric from R1. All the routers should have all the metrics with routers. Let's check R1's routing table

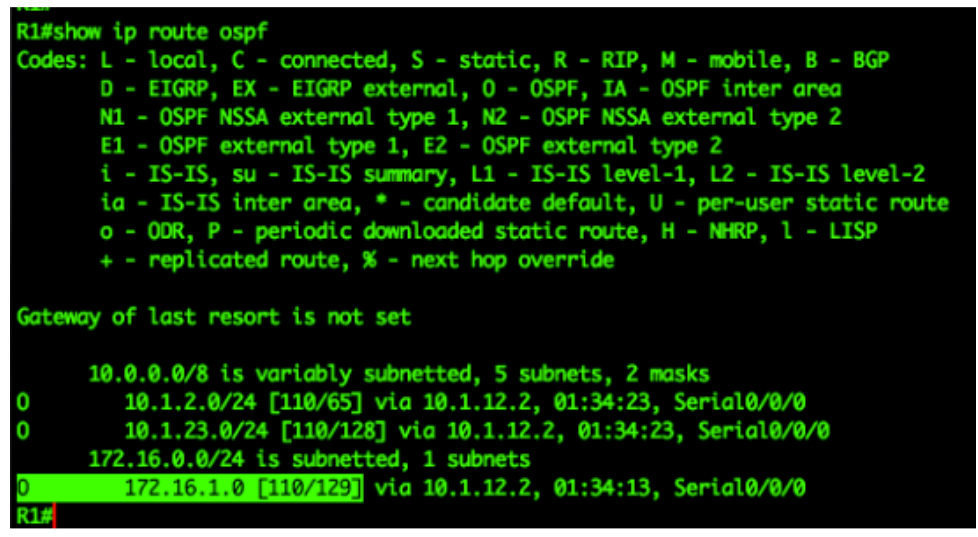

Figure 9: shows IP route 
Now, R1's routing table is showing that the cost for get 172.16.1.0/24 network is 129. Let's see how OSPF got the cost of 129. The interface from R1 to R2 is T1 link and the interface from R2 to R3 is T1. Each T1 link has a cost of 64 , so now we have two T1 links which make the cost as 128 plus the loopback interface cost, which is 172.16.1.0/24 Loopback interface has a cost of 1 $(128+1=129)$, and see the cost on R3.

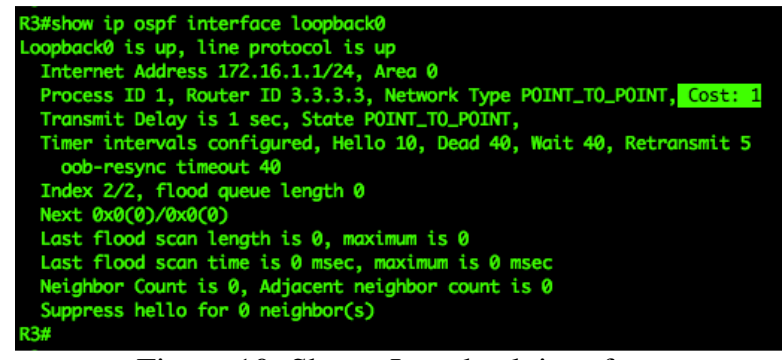

Figure 10: Shows Loopback interface

Let's change R3's serial 0/1 cost from 64 to 100 then check R1's routing table for the network 172.16.1.0/24 if the metric value has been changed or not.

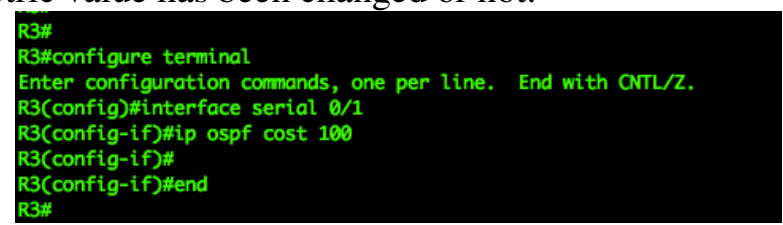

Figure 11: Assigning cost

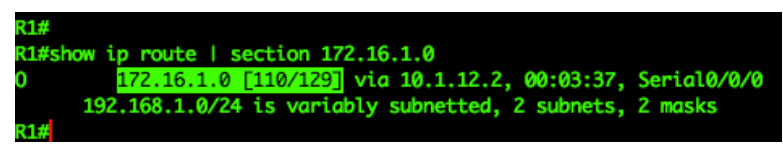

Figure 12: OSPF after assigning cost

After changing R3's interface serial0/1 cost to 100, R1 still sees the metric as 129 for the network 172.16.1.0/24. Nothing has been changed in R1. Let's check R1's routing table for changes after R2's serial0/1 cost from 64 to 100 .

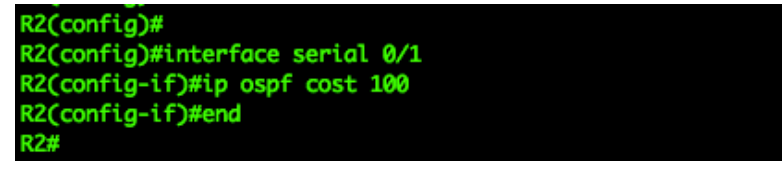

Figure 13: Assigning cost for Interface SE0/1

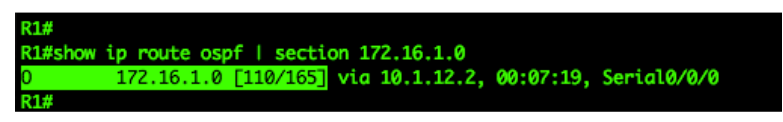

Figure 14: Show OSPF route

The metric went up from 129 to 165 . Now, we have to know which OSPF uses to calculate the metric. 
In summary, OSPF doesn't use the sending interface cost to calculate route metric, and we tested that by changing R3's serial0/1 interface cost to 100 . OSPF calculates the cost by using the receiving interfaces of the router in the network. Router R2 was calculated the metric by their serial0/1 cost (100) plus the neighbor's cost for all the routes then R2 will advertise those routes to its neighbors.

\section{Conclusion}

The main objective of the system focused on choosing the exact hardware requirements like(Routers, switches and Cables) for creating a different types of wide Area Network architecture by using the Open Shortest Path First and Border Gateway Protocol for creating inter-autonomous system and intra-autonomous system. We can obtain the optimal performance of the Internal and External WAN (Wide Area Network) by manipulating the metrics of the BGP (Border Gateway Protocol) and Open Shortest Path First (OSPF) such as cost, delay, throughput and bandwidth. We can also ensure the QoS (Quality of Service) of the networks.

\section{References}

[1] Neha Grang and Anuj Gupta, "Compare OSPF Routing Protocol with other Interior Gateway Routing Protocols", IJEBEA 13-147, Vol 1, pp.2-4, 2013

[2] Nikhil Hemant Bhagat," Border Gateway Protocol -A Best Performance Protocol When Used For External Routing than Internal Routing", Vol 1, pp.1-2, 2012

[3] Mohammad Nazrul Islam and Md. Ahsan Ullah Ashique, "Simulation Based EIGRP over OSPF", Vol 1,pp.4-5, 2010

[4] Christopher Carothers, Murat Yuksel, David Bauer and Shivkumar Kalyanaraman, "A Case Study in Understanding OSPF and BGP Interactions Using Efficient Experiment Design”, Vol 1,pp.2-4, 2006

[5] Toni Farley, Patrick Mcdaniel and Kevin Butlera, "Survey of BGP Security Issues and Solutions",AT\&T Labs Research, ACM Journal, Vol. V, Month 20YY

[6] Craig Labovitz, Farnam Jahanian and G. Robert Malan "Internet Routing Instability", IEEE/ACM Transactions on Networking, vol. 6, October ,pp.2-3, 1998

[7] Tony Larsson, Nicklas Hedman and Per Johansson, "Scenario-based Performance Analysis of Routing",Volume1,pp.3-4

[8] Toshihiko Kato,"Analysis of Internet Multicast Traffic Performance Considering Multicast Routing Protocol", Vol 1,pp.2-3

[9] http://en.wikipedia.org/wiki/Border_Gateway_Protocol

[10] http://features.techworld.com/networking/600/bgp-metrics/

[11] http://www.cisco.com/en/US/tech/tk365/technologies_tech_note09186a0080094431.shtml

[12] http://networkshinobi.wordpress.com/2011/11/19/ospf-metric-calculation-but-which-interface-doesit-use-to-calculate-its-metric/

[13] http://www.cisco.com/en/US/tech/tk365/tk480/tsd_technology_support_sub-protocol_home.html

[14] http://en.wikipedia.org/wiki/Link-state_advertisement

[15] http://en.wikipedia.org/wiki/Open_Shortest_Path_First 